Review

\title{
The Application of Lateral Flow Immunoassay in Point of Care Testing: A Review
}

\author{
Kan Wang $^{1,2}$, Weijian Qin ${ }^{1}$, Yafei Hou ${ }^{1}$, Kun Xiao $^{1}$,Wenqiang Yan $^{1}$ \\ ${ }^{1}$ Department of Instrument Science and Engineering, School of Electronic Information and Electrical Engineering, Shanghai Jiao \\ Tong University, Shanghai 200240, China. \\ ${ }^{2}$ Shanghai Engineering Research Center for Intelligent diagnosis and treatment instrument, Shanghai 200240, China. \\ Corresponding author. E-mail: wk_xa@sjtu.edu.cn
}

Received: Sep. 18, 2016; Accepted: Sep. 20, 2016; Published: Sep. 27, 2016.

Citation: Kan Wang, Weijian Qin, Yafei Hou, Kun Xiao and Yanwen Qiang, The Application of Lateral Flow Immunoassay in Point of Care Testing: A Review. Nano Biomed. Eng., 2016, 8(3): 172-183.

DOI: 10.5101/nbe.v8i3.p172-183.

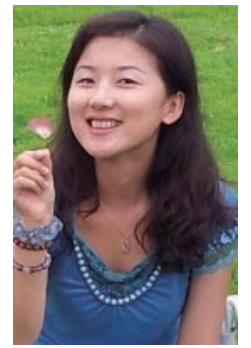

Kan Wang was born in 1979 and obtained her Ph.D. in biomedical and engineering from The Forth Military Medical University in 2008. After a postdoctoral fellowship at Shanghai Jiao Tong University in 2008, she was appointed as associate researcher of School of Electronic Information and Electrical Engineering, Shanghai Jiao Tong University in 2016. Her research interests cover nanotechnology development of early cancer detection and screening equipment, nonmaterial molecular imaging and biocompatibility evaluation, and biological tissue impedance spectrum characteristics.

\begin{abstract}
Point-of-care testing (POCT) is essential for providing a rapid diagnostic result in a prompt onsite diagnosis and treatment. A quick analysis time and a high sensitivity, with a sample-to-answer format, are the most important features for current POCT diagnostic systems. This review covers recent advances in POCT technologies with an emphasis on demonstrated and commercially available POCT diagnostic systems with laboratory quality using lateral flow immunoassay (LFIA). The system includes the integration of nanoparticles (NPs) in lateral flow test strips (LFTSs) and the mechanism through which particles improve the analytical performance of the fabricated strips. Several examples of NP-based LFTSs were selected to illustrate novel concepts or devices with promising applications as screening tools and superior alternatives to existing conventional strategies in clinical analysis, food safety, and environmental monitoring. In each analyte category, detection methods, configuration of LFIA modules, and advantages of POCT systems are reviewed and discussed along with future prospects. This review also discusses novel signal-enhancement strategies, optimal reader systems, and multiplex design prototypes, which have been employed for highly sensitive multiplex assay of LFTSs.
\end{abstract}

Keywords: Lateral flow immunoassay (LFIA); Point-of-care testing (POCT); Nanoparticles (NPs)

\section{Introduction}

Point of care test (POCT) are prevalent in the clinical field, where the goal of POCT is to develop a miniaturized, portable, intelligent self-contained system to assay biomarkers using complex samples. 
The lateral flow immunoassay (LFIA) is considered to be one of the most commercially feasible analytical tools for POCT, where it can perform a medical test quickly at the patient's location. Another benefit is that the test can be performed without sample pretreatment or the need for trained personnel and a laboratory. The user simply requires a drop of the sample and the process is driven via capillary force for fluid transport and delivery without external power. Commercial applications of LFIA mainly use colloidal gold as labels for diagnostics in food quality monitoring, drug testing, and environmental monitoring, such as detecting Enterobacteriaceae in food and water [1], medroxyprogesterone acetate residues [2], carbon black in a detection system for Dengue fever [3], and the detection of human chorionic gonadotropin in pregnancy testing [4]. This system exploits the color characteristics of markers and chromatographic separation to allow qualitative and semi-quantitative detection by the naked eye. However, these methods cannot satisfy clinical requirements because accurate quantitative detection is needed. At present, fluorescent lateral flow test strips (LFTSs) are undergoing accelerated development, where the intensity of the response can be detected quantitatively using appropriate test equipment. LFIA based on enzymecatalyzed chemiluminescence detection has been used for analyzing trinitrotoluene in forensic and environmental applications [5], as well as for detecting $\mathrm{Cry} 1 \mathrm{Ab}$ with an amplifier signal value [6]. LFIA based on quantum dots (QDs) has been developed for the detection of tumor markers $[7,8]$, mercury ions $(\mathrm{Hu}$ et al. 2014), avian influenza virus [9], pesticides and nerve agents [10].

This review focuses on the application of LFIA in POCT, including the integration of nanoparticles (NPs) in LFTSs and the mechanism through which particles improve the analytical performance of the fabricated strips. This review also discusses novel signal-enhancement strategies, optimal reader systems, and multiplex design prototypes, which have been employed for highly sensitive multiplex assay of LFTSs. This review selects several examples of NPbased LFTSs to illustrate novel concepts or devices with promising applications as screening tools and superior alternatives to existing conventional strategies in clinical analysis, food safety, and environmental monitoring.

\section{Applications of the Lateral Flow Immunoassay}

LFIA keeps the advantage of the special binding between antigen and antibody in immune test technology. Even though the technology takes the immune reaction on the glass fiber and nitrocellulose membrane without departing the binding markers and markers free, the method eliminates the tedious pipetting, washing step. Therefore, its operation is simple and fast (under 20 min getting the result) and the staff don't need training, even without any equipment or just with simple devices. It is very suitable for field testing and now widely used in a variety of fields of medicine, agriculture, animal husbandry and veterinary, environmental, food and supervision.

\section{Application in the medicine field}

Immunochromatographic analysis technology is firstly used to test the human chorionic gonadotropin (HCG) and this approach has been very mature and widely used in women pregnancy testing. In clinical diagnosis, immunochromatographic analysis technology possesses a very important significance and is a typical POCT, which is fast and thus allows timely rescue of lives. In the immunochromatographic assay, myoglobin (Mb) [11], CK-MB [12], troponin I (cTnI) [13] and troponin T (cTnT) [14], etc. are often used to help diagnose AMI biochemical markers by docoters. Immunochromatographic analysis technology also gets widely attention in testing fecal blood field [15]. Clavijo, et al. [16] utilized immunochromatographic analysis technology to test Brucella specific antibody in serum, through experimental comparison, the detection sensitivity was higher than traditional ELSIA detection method.

\section{Detection of pathogenic microorganisms}

Takeda utilized the immune colloidal gold chromatography strip to directly test the E. coli in the water sample, to compare ELISA with the concordance rate of $87.2 \%$, and to compare the culture method with the concordance rate of $89.10 \%$. The chromatography cost 5 minutes, which was shorter than any other test method. In addition, the immunochromatography test technology was also used to detect pathogens: Vibrio cholerae, adenovirus, hepatitis B surface antigen, and $\mathrm{H}$. pylori antigens, ect. Immunochromatographic 
techniques can detect microbial indicators of various suspicious for patients easily, quickly and with a low cost.

\section{Detection of human hormone or related proteins}

Since the first use in testing HCG, researchers have developed many different immunechromatography system to detect different hormones and protein targets. For example, Osikwicz utilized colloidal selenium to test HCG; Hedstrom utilized the immunechromatography to test urine trypsinogen II of patients with acute pancreatitis. Li utilized avidin-biotin immunechromatography technique to detect the cTnI. Immunechromatography technique of disease-related proteins comparing with the previous chemiluminescent or other means of detection have greatly improved the detection rate which is very important for timely diagnosis and treatment.

\section{Parasitic disease diagnosis}

Many parasitic diseases bear epidemic, highly infectious and other characteristics. There is a great need for simple, rapid, efficient, inexpensive diagnostic methods used on a massive scale. Thus, the immune chromatographic techniques in the diagnosis of parasitic diseases has also been increasingly widely used. Currently, for plasmodium, schistosomiasis, leishmaniasis, amoebiasis, filariasis, trypanosomiasis, cryptosporidiosis [17] immunochromatographic diagnostic methods have been established.

\section{Blood and urine drug monitoring}

Buecher using colloidal gold as a label has detected in the urine of illicit drugs (opiate, barbiturate, THC, amphetamine, cocaine, benzodiazepine, PC). Detection sensitivity and specificity of that test method is very consistent with gas chromatography/mass spectrometry (GC/MS) and EMIT (enzyme multiplied immunoassay technique).

Immunochromatographic analysis technology for application has widely researched in our country. For example, Zhao, et al. utilized Chlamydia trachomatis immunochromatographic strip to monitor the Chlamydia trachomatis (cT), UU (uu) and other infections incidence and to analyze their sensitivity and resistance to antimicrobial drugs; $\mathrm{Li}$, et al. [18] have researched the application of Immunochromatographic analysis technology in rapid detection of influenza viruses. In addition, hepatitis B surface antigen [19], Brucella [20] and Helicobacter pylori antigens [21] and other pathogens detection are also the hot researcher spot of immunochromatography technology. Yang Yonghong [22] etc utilized mycobacterium secreted protein antigen by using nitrocellulose membranecoated to detect serum and pleural effusion TB antibody.

\section{Drug detection}

Worldwide prevalence of drugs is increasingly becoming an important factor threatening people's health. Immunochromatographic detection of drugs have been widely used, such as cocaine, marijuana, coffee, heroin and other aspects, and there are already many commercialized drug testing kits. The recent quantitative detection method of drug is accurate, but the instrument is expensive and need strict experimental condition. Researchers at home and abroad are both looking for a simple, fast and accurate testing method. Gao Yueming et al. developed an immune colloidal gold based rapid drug quantitative testing method. For the content of some material in the human body constently change within a certain range, the results only showed positive or negative, which was unsatisfactory. Some researchers succeed in quantitative detection of theophylline and cholesterol in blood which based on enzyme colorimetric and realized by comparing chromogenic height on test strips with standard curve. Both clinical medicine, veterinary drug residue and drug detection are in urgent need of realizing simple and rapid quantitative detection.

\section{Live animal quarantine, meat distribution process monitoring, and testing of pesticides or veterinary drugs residues in food}

Immunochromatographic method is widely used in antigen or antibody detection in animal quarantine, such as swine fever, PRRS, parvovirus, infectious bursal disease, Newcastle disease, rabies and other infectious pathogens. With the development of industrialization, more and more pesticides, hormones, antibiotics are used for crop cultivation and animal husbandry. The residues of harmful substances in food can seriously affect people's health and life. Because of the large circulation of food, it is imperative to use a simple, fast, and low cost detection method. Currently immunochromatographic technology has been applied to a large number of food safety testing, such as 
detection of antibiotic residues, pesticide residue testing, hormone testing.

\section{Environmental detection}

For various reasons, there may be harmful microbes, hormones and other substances in the environment, and timely detection of suspect (water or air) is necessary. Currently there have been numerous studies on the environment immunochromatographic detection. There is a growing concern about the impact of environment changes to human health, those previously time-consuming testing methods will be increasingly replaced by simple and economical immunochromatographic method. So far, the immunochromatographic detection technology is applied to various areas of our lives. But immunochromatographic technology is not a panacea, there are many areas need to be developed and researched; research on immune chromatography technology in various application areas caused concern of many scholars, business and ongoing innovative research, so that this technology can be used to serve human life better.

\section{Research on the Detection System}

The development of equipment is processing widely about immune chromatography that can be used to achieve the purpose of qualitative detection and improve the accuracy of test and standard operation. Complete detection system can aid operator to get more accurate results and avoid interference of environment. Compared with the literature on ways of immune chromatography detection, the article of concerned device is fewer than the former. Qualitative test equipment developed by German ESE company is illustrated in Fig. 1. And the device provided stimulated luminescence can observe the emission light of a fluorescent strip on the strip, but does $\mathrm{n}$ ot have the function of quantitative detection [23].

Compared with commercial products of qualitative test equipment which is mainly based on the colloidal gold labeled test strip, detection about quantitative device is fewer both in domestic and abroad. A large number of companies have invested a lot of researches in the semi quantitative and quantitative detection of the Nano gold immune chromatography test, such as the United States of America's BBI, Europe's Mannheim Boehringer company and
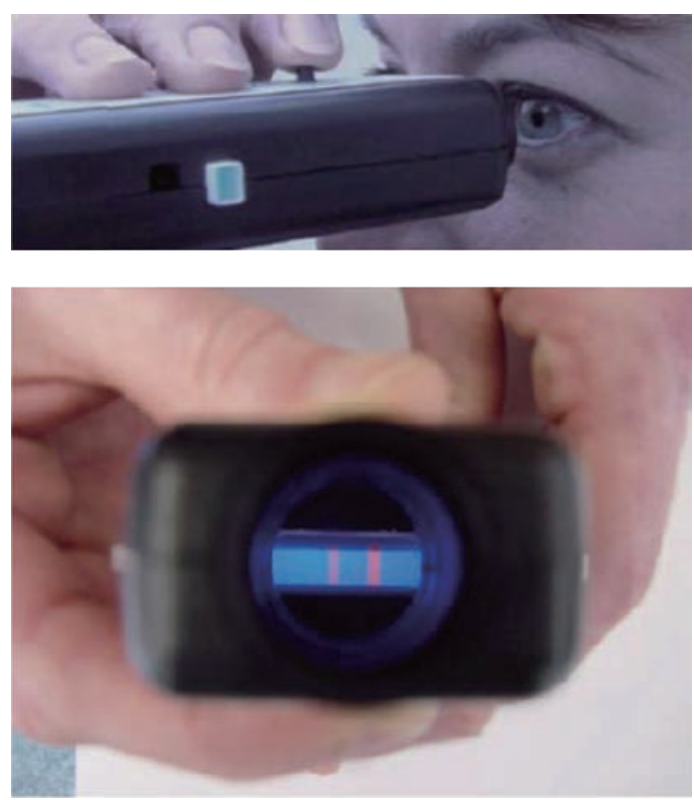

Fig. 1 Germany ESE test strip qualitative detection equipment [23].

Nycomed company. The test strip based on technology of Nano gold immune chromatography of Troponin and myoglobin about diagnosing acute myocardial infarction was launched by Boehringer Mannheim company. Simple measure devices being used to match these strip had passed through clinical testing. The precision and accuracy were consistent with the requirements of quantitative determination [24]. Chandler, et al. [25] realized quantitative detection about Nano gold immune chromatography test strip through the concentration of ladder type being displayed in the test paper and then the color signal of the strip being converted to digital signal by a portable instrument. A CD-RO optical detector was designed by Suhyeon, et al. [26] for the quantitative detection of HBV, and the mechanical components and optical probe of the detector are illustrated in Fig. 2. These researchers realized a cheap and fast detective system via the test sample transmitted by the motor. Portable Equipment used to Ultra-sensitively detect for protein markers and invented by $\mathrm{Li}$, et al. [27] made quantum dots to be achieved on the test strip quantitative detection that Nitrification ceruloplasmin detection range of is up to $1 \mathrm{ng} / \mathrm{mL}$. Zou, et al. [28] used system of quantum dot notation to achieve a quantitative detection of trichloropyridinol.

MagnaBioSciences company developed MAR magnetic strip for detection of the immune system. It is a huge upgrade and challenge to the traditional Nano-gold-labeled or labeled latex particles such as 


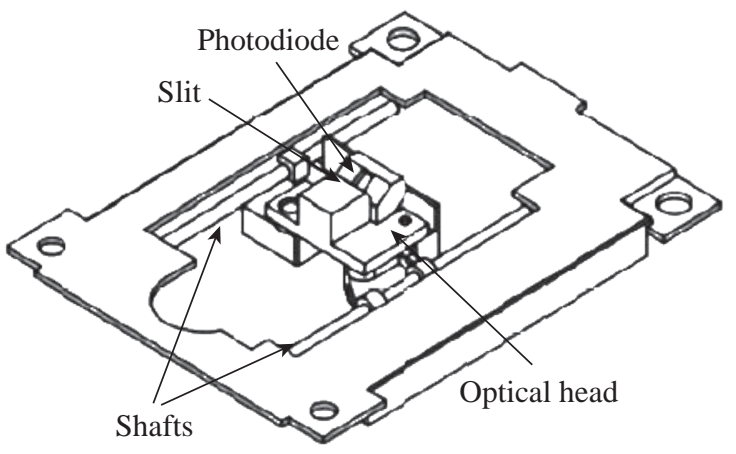

(a)

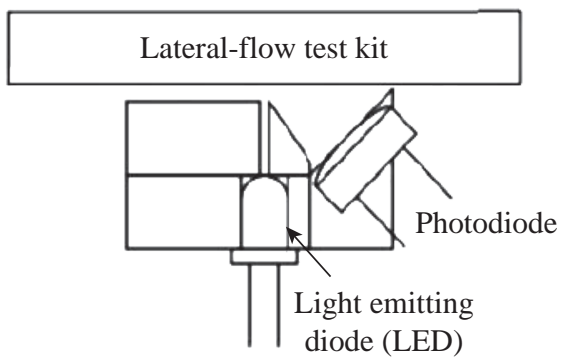

(b)

Fig. 2 (a) Detector; and (b) Optical part [26].

immunechromatographic strip [29]. The detection system (Fig. 3) directly tests strip in the corresponding three-dimensional magnetic marker area magnetization to get higher detection sensitivity than traditional methods via magnetizing target molecules of $60 \sim 380$ nm superparamagnetic Nano-magnetic beads mark by oscillating magnetic field. The device has received a lot of attention since the advent, and a large number of applications based on the detection of the detection system has been published in vitro. Because the method is different from the traditional method based on the color depth discrimination that is easy to be interfered by other colored material in the sample and the sensitivity is high [30-34, 39].

The quantitative study of the immune system started late in China, and it mainly concentrated on the quantitative detection of gold Nano gold immune chromatography test strip. Chinese Academy of Sciences, Guangzhou Institute of biomedical and Health Research Institute made immune layer technology apply to the detection of DNA binding protein, and biological sensor of using gold nanoparticles can be completed within 10 minutes of

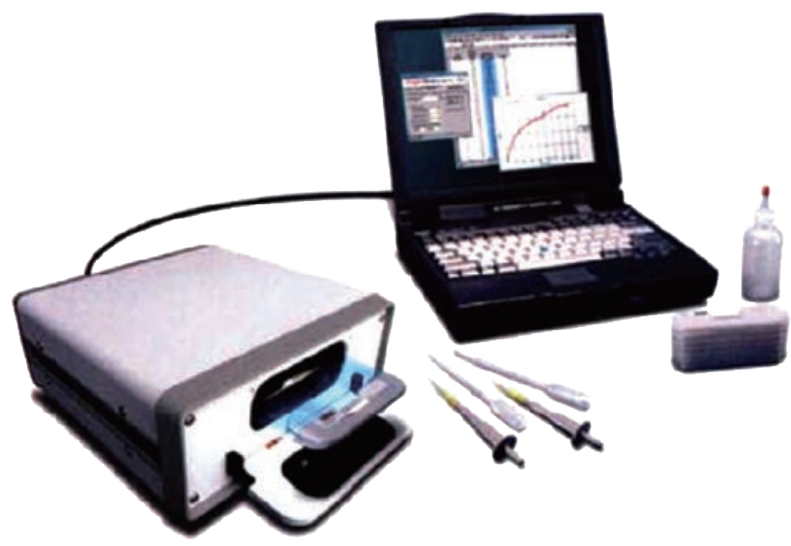

Fig. 3 Immune magnetic strip detection system MAR [29]. rapid detection [35]. The embedded reading instrument (Fig. 4) developed by Nankai University has high resolution CMOS image acquisition, and the detection accuracy is $1 \mathrm{ng} / \mathrm{mL}$, the variation coefficient $(\mathrm{CV})$ is less than $3 \%[36]$.

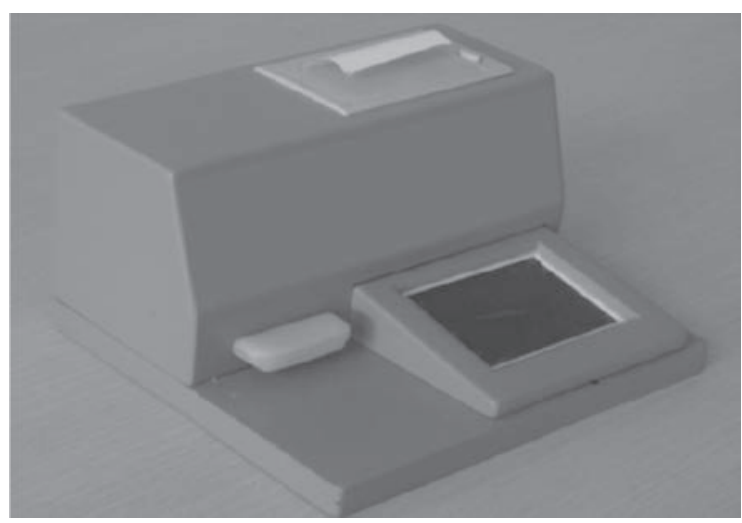

Fig. 4 Embedded detection system [36].

CAS Institute of Chemistry detected pesticide residues by using magnetic Nanoparticles. Determination of chromatography strip optical density and scales for oxygen concentration of the corresponding curve detection limit is $1.7 \mathrm{mg} / \mathrm{mL}$ [37] (Fig. 5). Shanghai Institute of Optical Precision Machinery arrived the purpose of quantitative detection of the gold tag strip through reflection photometer. They deduced the quantitative detection of CTnI equation. Correlation coefficient of the measured sample concentration and optical detection value is 0.989 . The variation coefficient is less than $5 \%$ as shown in Fig. 6 for the reflex photometer structure [38].

Fuzhou University developed testing equipment of HCG based on CCD colloidal gold marked strip. They got correlation coefficient is up to 0.9975 and 0.9914 [39] respectively through the two groups of 


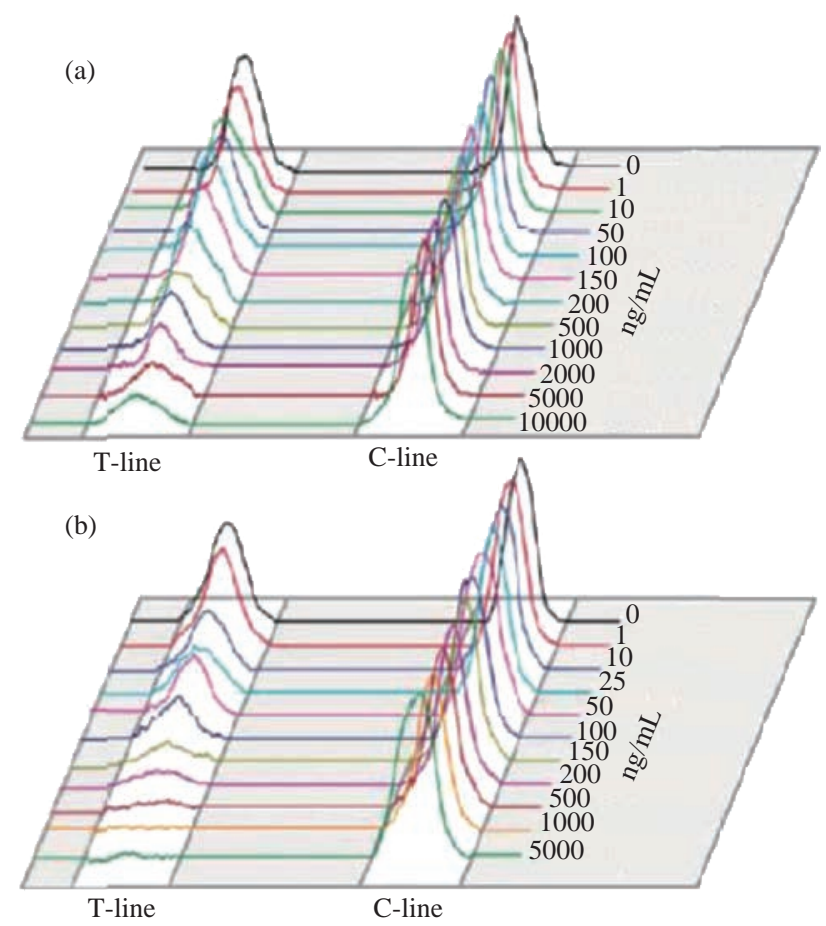

Fig. 5 The corresponding relationship between different concentrations of oxygen and phosphorus methyl and optical density [37]. different method about concentration of sample and the detection of optical density value. Supporting quantitative detection equipment about the magnetic strip and the quantum dots strip is few in domestic. Shanghai Jiao Tong University developed the testing equipment based on CCD technology of quantum dots immune chromatography test paper [40-43]. The device has realized early gastric cancer detection, such as the CagA, urease and quantitative detection, CEA, CA724 and other markers and obtained the medical device registration certificate (Fig. 7).

At the same time, they developed a portable immune layer chip detector based on embedded technology (Fig. 8) [44]. The system based on confocal imaging principle achieved a more accurate data collection and analysis for quantum dots immune chromatography test paper article on the fluorescent signal through the high performance LED point light source, low noise and high precision of photoelectric diode and mechanical movement module, positioning module. The single test time is about $1.5 \sim 2$ minutes, with

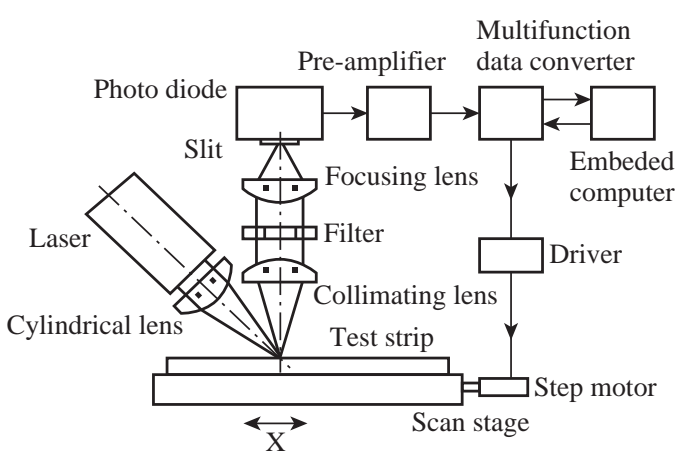

(a)

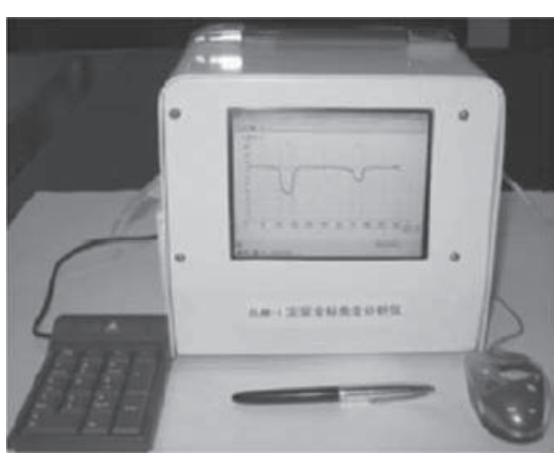

(b)

Fig. 6 Structure diagram of reflected light [38].

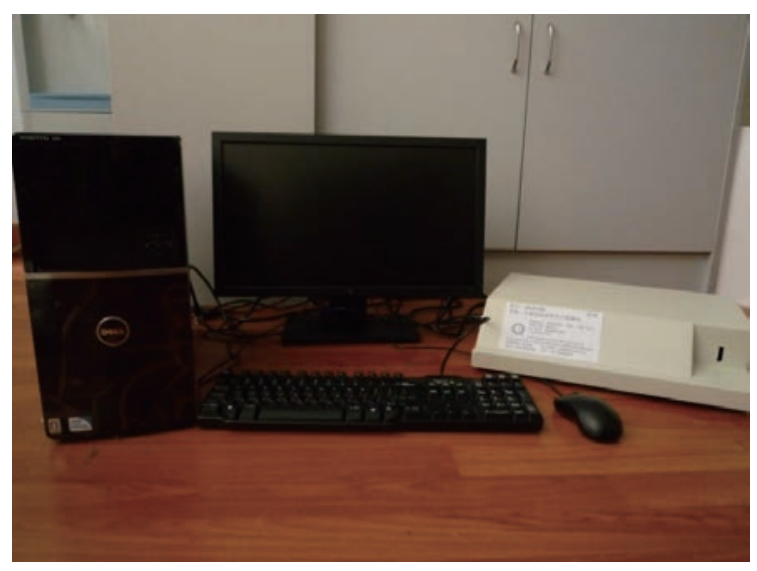

Fig. 7 Immune chromatography chip detector [40]. the fluorescent sensitivity of 0.4 molecular $/ \mu \mathrm{m}^{2}$, and the spatial resolution is $1000 \mu \mathrm{m}$. The application of embedded system greatly reduces the equipment volume and increase the portability. The system can generte test results more rapidly, save more effective treatment time, and thus save the patient>s life in the process of major disease rescue. Portable devices can avoid the defects of large equipment, and allow people to do basic preventive screening at home. Hence, the whole equipment is more suitable for the bed testing requirements in the community survey.

Recently, smartphone-based dual-modality imaging system [45] was developed by us, which could 

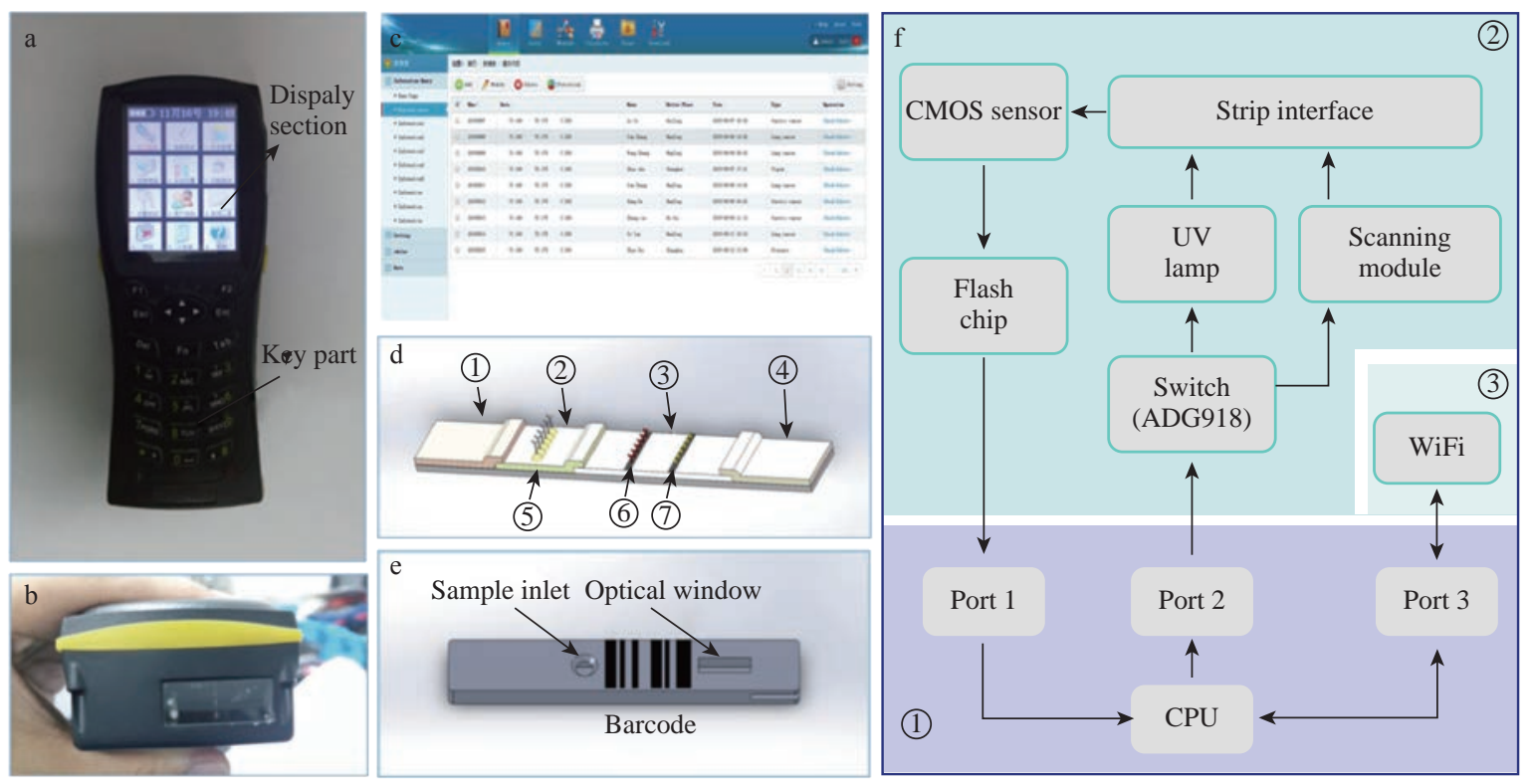

Fig. 8 Portable biological immune chromatography detector [44].

quantitativly detect color or fluorescent lateral flow test strips and be operated anywhere at any time, Fig. 9. The white and ultraviolet light of optical system was designed and could be changed according to the different kinds of strips. The Sobel operator algorithm was used in the software, which improved greatly the ability to distinguish the test area IURP वMKH background boundary information. \% पHxtracting the components from the Red, Green and Blue formatVof the color strips or only Red format of the fluorescent strips, it achieved high-signal intensity and sensitivity. Fifty samples were used to evaluate the accuracy of this system respectively; the ideal detection limit of the system were calculated separately from detection of HCG and CEA, and then kept the high stability. The results suggested that smartphonecontrolled dual-modality imaging system for the nextgeneration of portable and cost-effective platform provided different kinds of POC diagnosis.

\section{Research on Improving Sensitivity}

The improvement of detection sensitivity is mainly through changing the markers, adding color enhancement agent, amplifying immune response, integration of different NPs and so on. Currently, the analytical sensitivity and/or limit of detection (LOD) of LFICS can be mainly enhanced by using three types of NPs as alternative labels. (Fig. 10) These types include colored [e.g., GNPs, carbon NPs(CNPs), and colloidal selenium NPs (SNPs)], luminescent [e.g., quantum dots (QDs), up-converting phosphor NPs(UCPs), and dye-doped NPs], and magnetic NPs (MNPs) [46-48]. Horton et al. [48] used liquid sliver to strengthen the dyeing result after the colloidal gold was captured by the test line when testing mouse IgG. The sensitivity increased by 100 times. Muler et al. introduced avidin and biotin to immunochromatography system to detect fetal cardiac troponin T. One avidin has four binding sites which can bind with the biotin. So the signal intensity could be amplified greatly. Some researchers utilized lipidosome as labels which offer higher sensitivity owning to its strong plasticity [50], ability to set multiple antigens and antibodies in the membrane and to contain plenty of indicator in water phase space inside the membrane. Gred, et al. used europium microparticles as labels in immunochromatography system. The concentration of eosinophilic and neutrophils in blood can be reflected by the lipocalin in eosinophilic and neutrophils. Magnetic nanoparticals, as a new material developed in recent years, have a higher sensitivity in immunochromatographic assay through testing magnetism than ordinary luminescence method [51].

\section{Development of multivariate analysis}

In some cases, we need to test a variety of index to make a comprehensive judgement. So immunochromatographic assay is also developing head for the direction of multivariate analysis. Analyzing several substances in one strip which could not only improve detection efficiency but also 

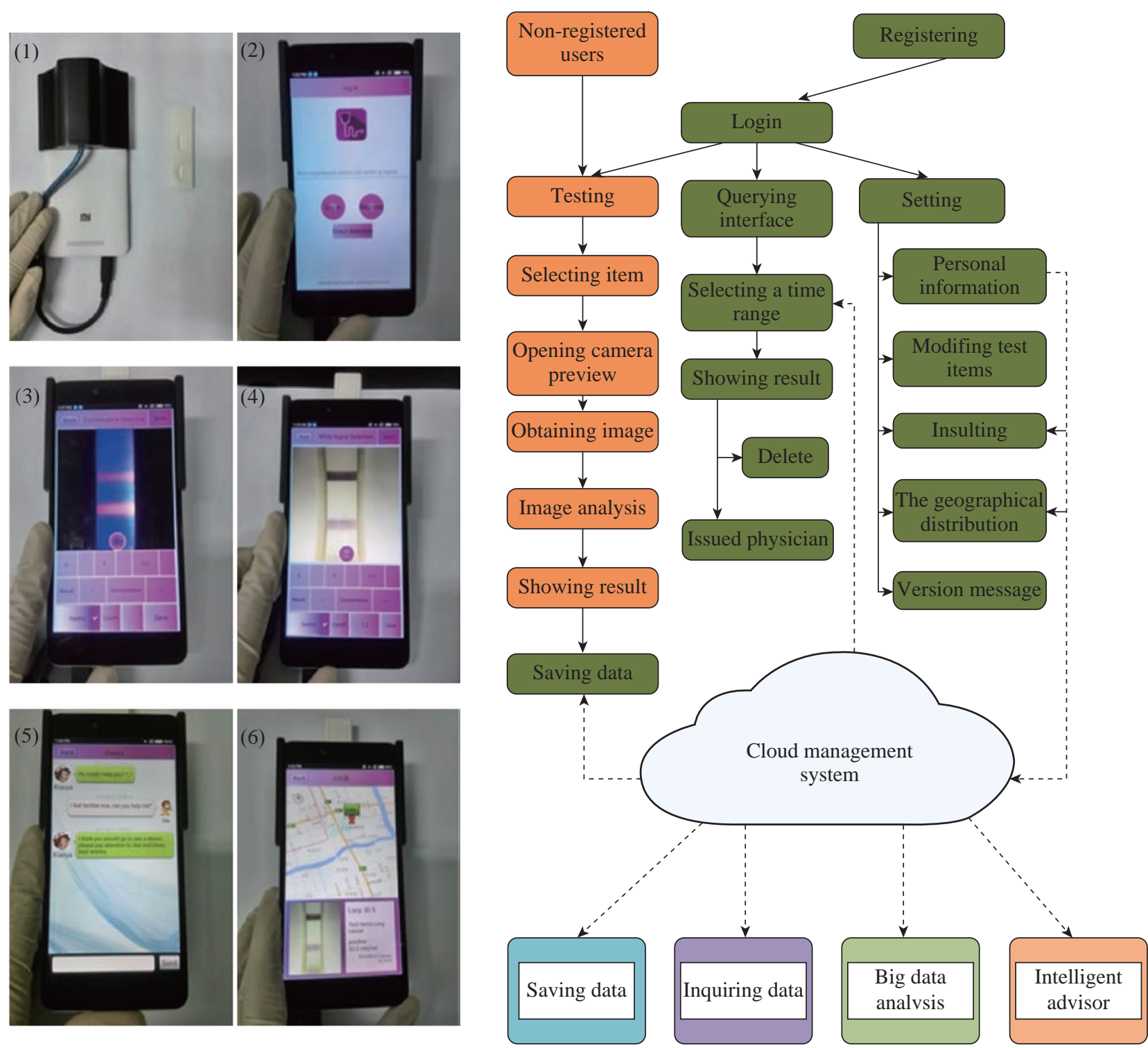

(a)

(b)

Fig. 9 (a) (1) Back view of testing equipment and the strip shell; (2) The main menu of the software; (3) The fluorescent strip testing displaying (CEA); (4) The color strip testing displaying (Colloidal strip for testing HCG); (5) The robot service through chat; (6) The location distribution of the test result in map. (b) Flow diagram of the device software [45].

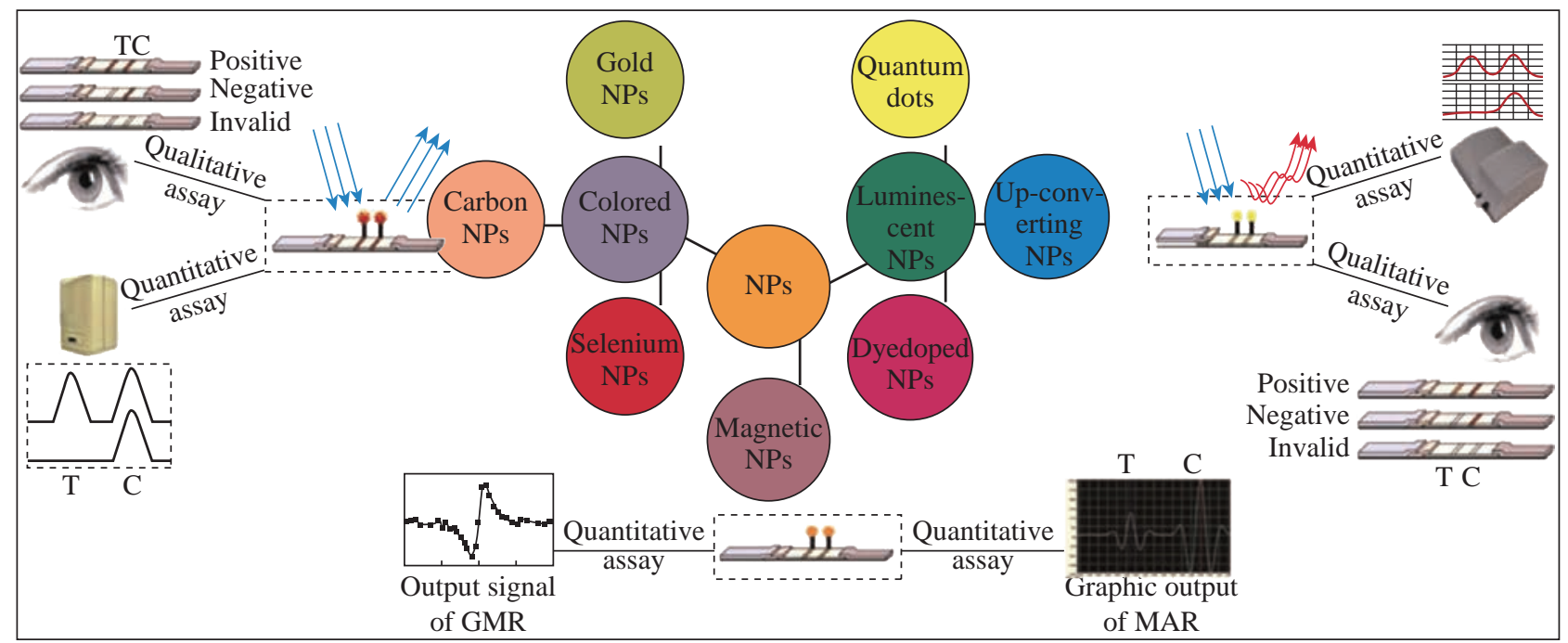

Fig. 10 Schematic illustration for various ultrasensitive LFICS detection from qualitative to quantitative [46]. 
lower testing costs. Besides, it had high application value for testing index which was well suited for multivariate analysis. For example, hepatitis B could be diagnosed by testing PreS1, PreS2, HBsAg, HbeAg and others. A variety of hormones, allergens and cytokines could be analyzed according to different testing requirements. Tjitra, et al., using immunochromatographic assay, developed a testing strip which could simultaneously analyze plasmodium falciparum and plasmodium vivax; it had good sensitivity, specificity and clinical application value. In Fig. 11, Buechler, et al. utlized one membrane sprayed with several $\mathrm{T}$ lines and succeeded in the simultaneous detection of seven forbidden drugs in urine [51]. Sterling, et al. employed different membranes and enzymes in different places of the membrane to separate and make the analyte visualized which could simultaneously test hemoglobin, glucose and cholesterol in blood. In addition, to detect multiple samples simultaneously, the traditional method was modified by designing several test lines in a single strip as described earlier. Corstjens, et al. developed a multiplexed UCP-based LFICS for the detection of antibodies against Mycobacterium tuberculosis, hepatitis $\mathrm{C}$ virus and human immunodeficiency virus [53]. An interesting device

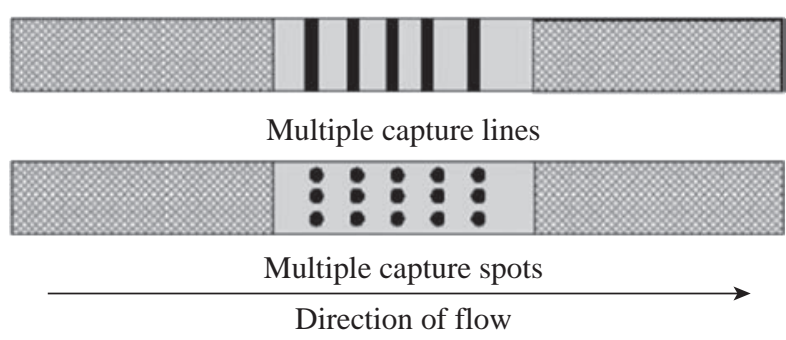

Fig. 11 Principle of multiplex lateral flow immunoassay [52].

with a 10 channel LFICS utilizing UCPs technology was also employed for the simultaneous detection of different antibodies against Yersinia pestis [54].

\section{Development from qualitative to quantitative detection}

Immunochromatographic assay has the characters of easy operation, rapid, specific and clear result, easy to judge and save, and no need of any instrument. As shown in Fig. $12 \& 13$, from top to bottom are masculine, feminine and invalid test results. Obviously, qualitative detection is insufficient in many occasions. Many test strips can only qualitatively analyze whether female is pregnant or not, but threatened abortion, ectopia, drug drainage, some obstetrical and gynecological diseases and postoperation treatment all need tracking the quantitative change of hCG and quantitative detection of hCG. Recently, there are

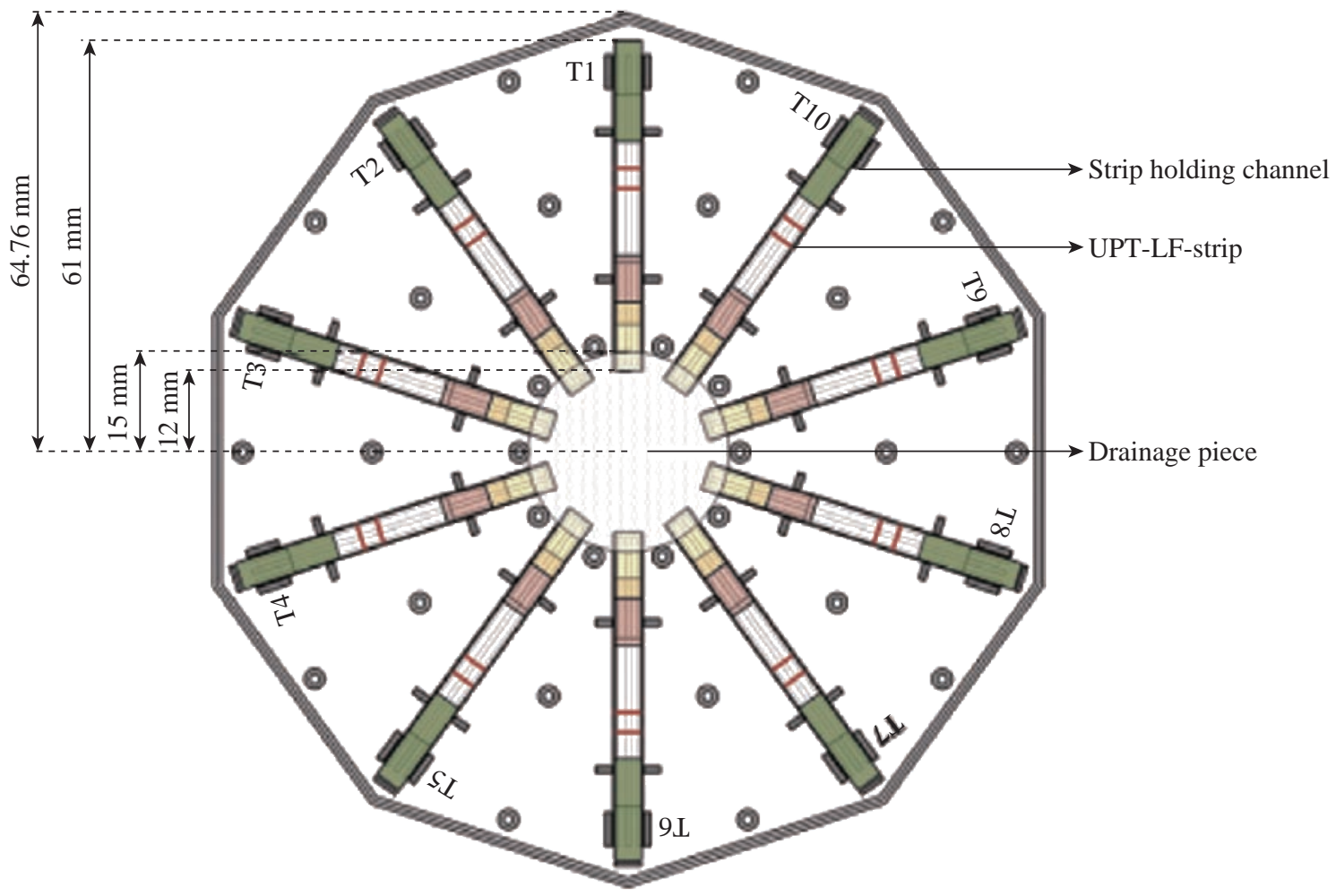

Fig. 12 The sketch map of 10 channel LFICS based on UCP [54]. 


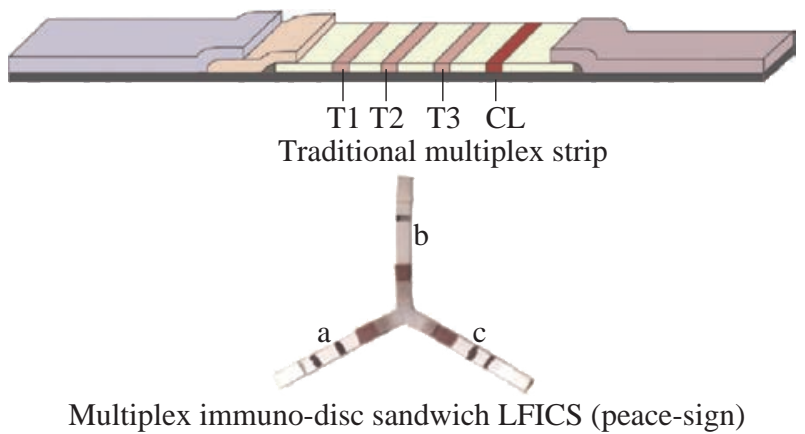

Fig. 13 Schematici llustration of multiplex detection model: traditional LFICS and "peace-sign" LFICS and results for detection of Staphylococcus aureus and Pseudomonas aeruginosa using (a) Staphylococcus aureus; (b) Pseudomonas aeruginosa; and (c) Staphylococcus aureus+Pseudomonas aeruginosa.[55].

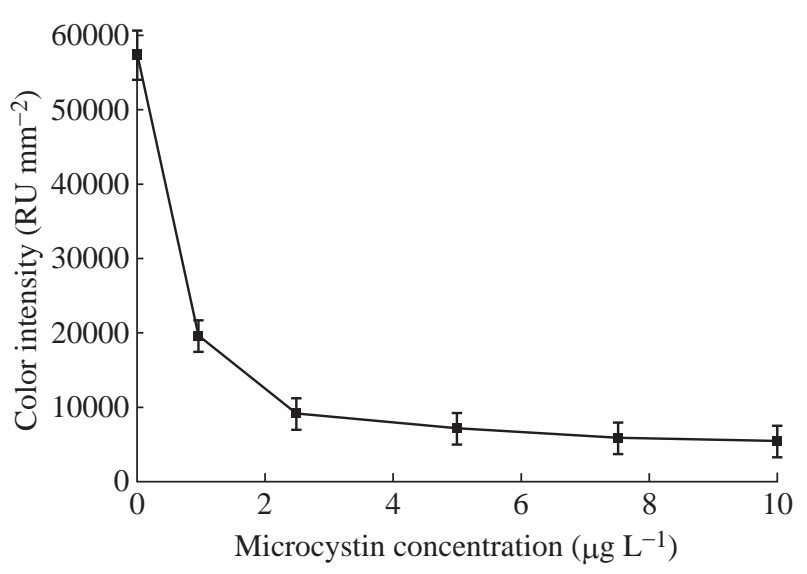

Fig. 14 The relation curve between color values of the strips and the concentrations of microcystin [56].

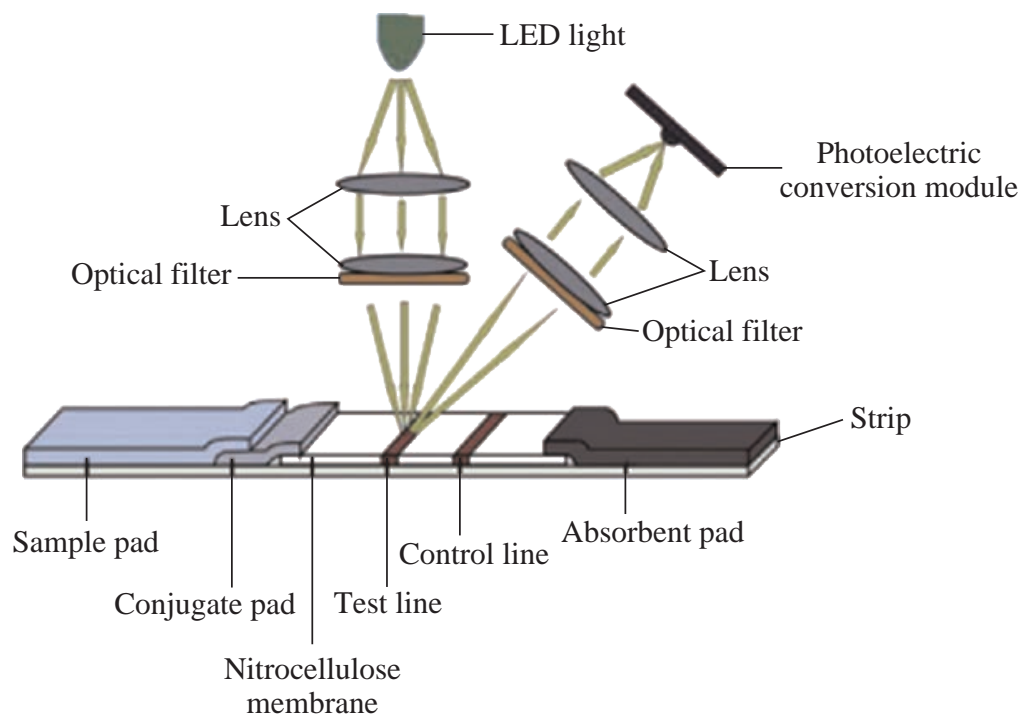

Fig. 15 Strip reader [57].

plenty of researches focusing on qualitative/quantitative immunochromatographic assay. Quantitative detection not only has significance in diagnosis of acute disease timely, but also is a very important method for the relevant quality inspection departments to perform health standards. Tippkötter, et al. [56] established a relation curve between color vaules of the strips and the concentration of the analytes in the rapid test of the concentration of microcystin. In the detection of clinical medicine, quick and easy quantitative detection could help doctors know the pathogenetic conditions of patients in time and implement the treatment, which has vital importance in treating acute diseases such as myocardial infarction. The realization of quantitative detection is not only based on the preparation of strips, but is also concerning the development of the detection instrument. At present, the main research interest on quantitative testing is using photoelectric detection method to transmit the optical signal to electrical signal and obtaining a digital signal, and thus to get the quantitative detection result. On the other hand, magnetic nanoparticles based LFTS could be analyzed through magnetic flux testing equipment to obtain quantitative results.

\section{Conclusions}

In general, LFICS is a rapid screening system used in various fields, such as medicine, food safety, and environmental monitoring, because of its outstanding advantages, including low cost, high speed, portability, and the lack of need for complicated equipment and 
technical expertise. Nevertheless, the performance of this system should be improved with respect to LOD or sensitivity, quantitative determination, and multiple/ simultaneous detection. Moreover, novel designs, such as the use of various NPs for LFICS, have been reported in recent literature. It also has a potential to accomplish complex diagnostic assays, e.g. nucleic acid short tandem repeat fingerprinting, as a sample to answer format for POCT by integrating all the functional modules into a microfluidic lab-on-a-chip. These advantages of the technology thus encourage extensive research efforts toward clinical diagnostic POCT for detecting various types of analytes such as proteins, cells, nucleic acids and metabolites.

\section{Acknowledgments}

This work was supported by the National Natural Science Foundation of China (Grant No. 81571835, 61503246, 81672247 and 81671737), National Key Basic Research Program (973 Project) (No.2015CB931802), the 863 High-Tech Project of China (No. 2014AA020700), and Shanghai Science and Technology Fund (No. 13NM1401500 and 15DZ2252000).

\section{References}

[1] J. Singh, S. Sharma, S. Nara, et al., Evaluation of gold nanoparticle based lateral flow assays for diagnosis of enterobacteriaceae members in food and water. Food Chemistry, 2015,170: 470-483.

[2] C. Song, A. Zhi, Q. Liu, et al., Rapid and sensitive detection of beta-agonists using a portable fluorescence biosensor based on fluorescent nanosilica and a lateral flow test strip. Biosensors \& Bioelectronics, 2013, 50: 6265 .

[3] C. Wang, F. Hou, Y. Ma, et al., Simultaneous quantitative detection of multiple tumor markers with a rapid and sensitive multicolor quantum dots based immunochromatographic test strip. Biosensors \& Bioelectronics, 2015, 68: 156-162.

[4] X. Yan, K. Wang, W. Lu, et al., CdSe/ZnS Quantum dot-labeled lateral flow strips for rapid and quantitative detection of gastric cancer carbohydrate antigen 72-4. Nanoscale Research Letters, 2016, 11(1): 138.

[5] H., Yang, Q., Guo, R., He, et al., A quick and parallel analytical method based on quantum dots labeling for ToRCH-related Antibodies. Nanoscale Research Letters, 2016, 4(12): 1469-1474.

[6] Q. Yang, X. Gong, T. Song, et al., Quantum dot-based immunochromatography test strip for rapid, quantitative and sensitive detection of alpha fetoprotein. Biosensors and Bioelectronics, 2011, 30(1): 145-150.

[7] Y. Yang, Y. Ma, H. Guan, et al., Phase coherence length in silicon photonic platform. Optics Express, 2015, 23(13): 16890-16902.

[8] D.J. You, T.S. Park, J.Y. Yoon, et al., Cell-phone-based measurement of TSH using Mie scatter optimized lateral flow assays. Biosensors \& Bioelectronics, 2013, 40(1):
180-185.

[9] H. Yun, Z. Chen, Y. Wang, et al., Polarizationrotating, Bragg-grating filters on silicon-on-insulator strip waveguides using asymmetric periodic corner corrugations. Optics Letters,2015, 40(23): 5578-5581.

[10] W. Zhang, X. Ge, Y. Tang, et al., Nanoparticle-based immunochromatographic test strip with fluorescent detector for quantification of phosphorylated acetylcholinesterase: an exposure biomarker of organophosphorus agents. The Analyst, 2013, 138(18): 5431.

[11] P. Ecollan, J.P. Collet, G. Boon, et al., Pre-hospital detection of acute myocardial infarction with ultrarapid human fatty acid-binding protein (H-FABP) immunoassay. International Journal of Cardiology, 2007, 119 (3): 349-354.

[12] J. Hampl, M. Hall, N.A. Mufti, et al., Upconverting phosphor reporters in immunochromatographic assays. Analytical Biochemistry, 2001, 288 (2): 176-187.

[13] F. Xu, H. Xu, H. Gu, et al., Development of lateral flow immunoassay system based on superparamagnetic nanobeads as labels for rapid quantitative detection of cardiac troponin I. Materials Science and Engineering: $C$, 2009, 29 (3): 702-707.

[14] T. Kurihara, A. Yanagida, H. Yokoi, et al., Evaluation of cardiac assays on a benchtop chemiluminescent enzyme immunoassay analyzer, PATHFAST. Analytical Biochemistry, 2008, 375 (1): 144-146.

[15] L. Jun, Y. Wenquan, W. Nanhua, et al., Analysis result of faecal occult blood test based on colloidal immunization method. Lab Med Clin, 2008 (2): 111-112

[16] E. Clavijo, R. Díaz, A. Anguita, et al., Comparison of a dipstick assay for detection of Brucella-specific immunoglobulin $\mathrm{M}$ antibodies with other tests for serodiagnosis of human brucellosis. Clinical and Vaccine Immunology, 2003, 10 (4): 612-615.

[17] Z. Ruiming, T. Dandan, C. Jie, et al., Detection of mycoplasma and chlamydia in 292 cases of female urogenital tract infections and the antibiotic susceptibility analysis. Chin Prac Med, 2008, 3(28): 15-16.

[18] Y. Li, H. Chen, P. Wang, et al., Application of gold immunochromatographic assay for a rapid detection of influenza viruses. Journal of the Fourth Military Medical University, 2008, 29 (18): 1652-1654.

[19] S. Lin, Y. Zhang, Optimization of production process of diagnostic reagent of colloidal gold for HBsAb. Progress in Modern Biomedicine, 2008, 8 (2): 289-292.

[20] J. Tang, X. Li, X. Wang, et al., Development of colloidal gold-immunochromatographic assay for brucellosis, Chin J Biol, 2007, 20 (2): 119-121.

[21] T.S. Chen, F.Y. Chang, S.D. Lee, et al., Serodiagnosis of Helicobacter pylori infection: comparison and correlation between enzyme-linked immunosorbent assay and rapid serological test results. Journal of Clinical Microbiology, 1997, 35 (1): 184.

[22] Y. Yang., Application of gold immunchromatographic assay in rapid diagnosis of tuberculosis. Gansu Science and Technology, 2009, 24 (22): 173-174.

[23] K. Faulstich, K. Haberstroh, R. Gruler, et al., Handheld and portable test systems for immunodiagnostics, nucleic acid detection and more. Society of PhotoOpticalInstrumentation Engineers, 2008, 6945 (1): 69450H1-69450H10.

[24] D. Ming, Study of nano-gold immunochromatographic quantitative assay based on photoelectric detection and processing. Journal of the Fuzhou University, 2005: 4-5.

[25] J. Chandler, T. Gurmin, N. Robinson, et al., The place of gold in rapid tests. IVD Technology, 2000, 6 (2): 37-49.

[26] S. Kim, J.K. Park, Development of a test strip reader for a lateral flow membrane-based immunochromatographic 
assay. Biotechnology and Bioprocess Engineering, 2004, 9(2): 127-131.

[27] Z. Li, Y. Wang, J. Wang, et al., Rapid and sensitive detection of protein biomarker using a portable fluorescence biosensor based on quantum dots and a lateral flow test strip. Analytical Chemistry, 2010, 82 (16): 7008-7014.

[28] Z. Zou, D. Du, J. Wang, et al., Quantum dot-based immunochromatographic fluorescent biosensor for biomonitoring trichloropyridinol, a biomarker of exposure to chlorpyrifos. Analytical Chemistry, 2010, 82 (12): 5125-5133.

[29] MagnaBioSciences, LLC (MBS). http://www. magnabiosciences.com/

[30] Y. Wang, H. Xu, M. Wei, et al., Study of superparamagnetic nanoparticles as labels in the quantitative lateral flow immunoassay. Materials Science and Engineering: C, 2009, 29 (3): 714-718.

[31] T.C. Granade, S. Workman, S.K. Wells, et al., Rapid detection and differentiation of antibodies to HIV1 and HIV-2 using multivalent antigens and magnetic immunochromatography testing. Clinical and Vaccine Immunology, 2010, 17 (6): 1034-1039.

[32] R.B. Peck, J. Schweizer, B.H. Weigl, et al., A magnetic immunochromatographic strip test for detection of human papillomavirus 16 E6. Clinical Chemistry, 2006, 52 (11): 2170-2172

[33] S. Workman, S.K. Wells, C.P. Pau, et al., Rapid detection of HIV-1 p24 antigen using magnetic immunochromatography (MICT). Journal of Virological Methods, 2009,160(1): 14-21.

[34] S. Handali, M. Klarman, A.N. Gaspard, et al., Development and evaluation of a magnetic immunochromatographic test to detect Taenia solium, which causes taeniasis and neurocysticercosis in humans. Clinical and Vaccine Immunology, 2010, 17(4): 631-637.

[35] Z. Fang, C. Ge, W. Zhang, et al., A lateral flow biosensor for rapid detection of DNA-binding protein c-jun. Biosensors and Bioelectronics, 2011, 21(1): 192-196.

[36] J. Mei, Q. Ye, W. Zhou, et al. Development and study of lateral flow test strip reader based on embedded system. IEEE: Electronic Measurement \& Instruments, 2011; 1,201-204.

[37] C. Liu, Q. Jia, C. Yang, et al., Lateral Flow Immunochromatographic assay for sensitive pesticide detection by using $\mathrm{Fe} 3 \mathrm{O} 4$ nanoparticle aggregates as color reagents. Analytical Chemistry, 2011, 83(17): 6748-6784.

[38] L. Huang, Y. Zhang, C. Xie, et al., Research of reflectance photometer based on optical absorption. Optik International Journal for Light and Electron Optics, 2010, 121(19): 1725-1728.

[39] Y. Li, N. Zeng, M. Du, Study on the methodology of quantitative gold immunochromatographic strip assay. IEEE: Intelligent Systems and Applications (ISA), 2010, 2 : $1-4$.

[40] X. Zhang, D. Li, C. Wang, et al., A CCD-based reader combined quantum dots-labeled lateral flow strips for ultrasensitive quantitative detection of anti-HBs antibody. Journal of Biomedical Nanotechnology, 2011, 8: 1-8.

[41] G. Chen, K. Wang, C. Li, et al., A CCD-based reader combined with CdS quantum dot-labeled lateral flow strips for ultrasensitive quantitative detection of CagA. Nanoscale Research Letters, 2014, 9: 57.

[42] Y. Zheng, K. Wang, J. Zhang, et al., Simultaneous quantitative detection of helicobacter pe456ylori based on a rapid and sensitive testing platform using quantu dotslabeled immunochromatiographic test strips. Nanoscale Research Letters, 2016, 11: 62.
[43] X. Zhang, H. Yang, K. Wang, Image processing for the ccd based lateral flow strip detector. Nano biomedicine engineering, 2010, 2(4): 214-217.

[44] W. Qin, K. Wang, K. Xiao, et al., Carcinoembryonic antigen detection with "handing"-controlled fluorescence spectroscopy using a color matrix for point-of-care applications. Biosensors and Bioelectronics, 2016, Under review.

[45] Y. Hou, K. Wang, K. Xiao, et al., Smartphone-based dualmodality imaging system for quantitative detection of color or fluorescent lateral flow immunochromatographic strips. Scientific Reports, 2016, Under review.

[46] H. Xiao, A. Zoraida, X. Heng, et al., Membranebased lateral flow immunochromatographic strip with nanoparticles as reporters for detection: A review. Biosensors and Bioelectronics, 2016, 75: 166-180.

[47] P. Chun, Colloidal gold and other labels for lateral flow immunoassays. In Lateral Flow Immunoassay. Humana Press, 2009: 75-93.

[48] K. Faulstich, R. Gruler, M. Eberhard, et al., Handheld and portable reader devices for lateral flow immunoassays. In Lateral Flow Immunoassay. Humana Press, 2009: 157-184.

[49] J. Horton, S. Swinburne and M. O'Sullivan, A novel, rapid, single-step immunochromatographic procedure for the detection of mouse immunoglobulin. Journal of Immunological Methods, 1991, 140 (1): 131-134.

[50] K.A. Edwards, A.J. Baeumner, Optimization of DNAtagged dye-encapsulating liposomes for lateral-flow assays based on sandwich hybridization. Analytical and Bioanalytical Chemistry, 2006, 386 (5): 1335-1343.

[51] S.K. Sharma, B.S. Eblen, R.L. Bull, et al., Evaluation of lateral-flow Clostridium botulinum neurotoxin detection kits for food analysis. Applied and Environmental Microbiology, 2005, 71 (7): 3935-3941.

[52] K.F. Buechler, S. Moi, B. Noar, et al., Simultaneous detection of seven drugs of abuse by the Triage panel for drugs of abuse. Clinical Chemistry, 1992, 38 (9): 16781684.

[53] P.L. Corstjens, Z. Chen, M. Zuiderwijk, et al., Rapid assay format for multiplex detection of humoral immune responses to infectious disease pathogens (HIV, HCV, and TB). Annals of the New York Academy of Science, 2007, 1098: 437-445.

[54] W. Hong, L. Huang, H. Wang, et al., Development of an up-converting phosphor technology-based 10-channel lateral flow assay for profiling antibodies against Yersinia pestis. Journal of Microbiological Methods, 2010, 83: $133-140$

[55] C.Z. Li, K. Vandenberg, S. Prabhulkar, et al., Paper based point-of-care testing disc for multiplex whole cell bacteria analysis. Biosensors and Bioelectronics, 2011, 26: 43424348.

[56] N. Tippkötter, H. Stückmann, S. Kroll, et al., A semiquantitative dipstick assay for microcystin. Analytical and Bioanalytical Chemistry, 2009, 394 (3): 863-869.

[57] C., Li, W., Luo, H., Xu, et al., Development of an immunochromatographic assay for rapid and quantitative detection of clenbuterol in swine urine. Food Control, 2013, 34: 725-732.

Copyright $(2016$ Kan Wang, Weijian Qin, Yafei Hou and Kun Xiao. This is an open-access article distributed under the terms of the Creative Commons Attribution License, which permits unrestricted use, distribution, and reproduction in any medium, provided the original author and source are credited. 